Copyright by the Acoustical Society of America. Ross, B. W., \& Burdisso, R. A. (1999). Low frequency passive noise control of a piston structure with a weak radiating cell. Journal of the Acoustical Society of America, 106(1), 226-232. doi: 10.1121/1.427051

\title{
Low frequency passive noise control of a piston structure with a weak radiating cell
}

\author{
Bradley W. Ross and Ricardo A. Burdisso \\ Vibration and Acoustic Laboratories, Department of Mechanical Engineering, Virginia Polytechnic Institute \\ and State University, Blacksburg, Virginia 24061-0238
}

(Received 15 April 1998; accepted for publication 31 March 1999)

\begin{abstract}
The concept of a weak sound radiating cell is proposed to reduce the low-frequency radiated noise from structures. The cell consists of two mechanically coupled surfaces such that, when placed on a vibrating structure, the response of the two surfaces are nearly out-of-phase and nearly of the same strength over a wide frequency range. This structure response leads the cell to behave as an acoustic dipole and thus a poor sound-radiating source. The control of low-frequency structurally radiated noise is then achieved by covering the structure with an array of these weak radiating cells, i.e., surface treatment. Thus the surface treatment essentially transforms the response of the structure to that of a distributed array of dipoles yielding a low sound radiating structure. A theoretical model of a single weak radiating cell applied to a simple piston structure was developed and experimental verification was performed. Overall sound power level reductions of over $6 \mathrm{~dB}$ were experimentally achieved between 400 and $1600 \mathrm{~Hz}$ with maximum reductions of over $30 \mathrm{~dB}$ at discrete frequencies. (C) 1999 Acoustical Society of America. [S0001-4966(99)02007-X]
\end{abstract}

PACS numbers: 43.50.Sr, 43.55.Fw [PJR]

\section{INTRODUCTION}

The cancellation of sound has become a major point of interest as a result of stiffened federal regulations and increased consumers' desires for a quieter product. In the past this has led to extensive research to gain a better understanding of the acoustic behavior of structures such as helicopters, ${ }^{1}$ airplanes, ${ }^{2}$ and power transformers. ${ }^{3}$ The attenuation of structurally radiated noise, in particular at low frequencies, from these and other systems has been intensively pursued. Traditional methods of noise control include applying passive treatments to the radiating structures such as structural damping and sound absorptive materials. However, absorptive materials in particular are not a practical means of attenuation at low frequencies because of the thickness requirement to absorb the large acoustic wavelengths. In light of this, researchers have investigated alternate passive control techniques designed to reduce the low-frequency sound radiation from structures. Attempts have been made to increase the transmission loss of panels by embedding arrays of acoustic resonators. ${ }^{4,5}$ The placement of optimally sized masses on a structure for modal restructuring has also been investigated. ${ }^{6}$ For almost a century now, passive tuned vibration absorbers (TVAs) have been investigated to impede tonal and broadband motion of a structure. ${ }^{7}$

Recently, active control has emerged as a viable technology to solve the problem of low-frequency noise radiated from structures. ${ }^{8}$ This has allowed for the traditional tuned vibration absorber technology to be extended to hybrid absorbers, e.g., combination of passive and active methods. ${ }^{9,10}$ The use of active control has also resulted in the concept of hybrid and active skins for radiating structures. ${ }^{11,12}$

More relevant to the research in this paper, recent work on active control has revealed that partitioning a structure or panel into individually actuated subpanels that cancel the local volume velocity is an effective approach for low- frequency sound reduction. ${ }^{13-17}$ The active volume velocity control approach uses actuators to reduce either the vibration of the subpanel or its radiation efficiency, e.g., modifying the response of the subpanel from a monopole to a dipole acoustic source. For example, in work performed by St. Pierre et al., a loudspeaker is embedded into a vibrating panel and used to control the sound radiated by minimizing the volume velocity. ${ }^{13}$ The panel and loudspeaker make up the components of the actively created dipole source. Unfortunately, the complexity, power requirement, additional weight, unreliability, and high cost of active control systems have significantly limited their practical application to realistic systems.

Here the concept of a weak radiating cell as a new lowfrequency passive noise control device is presented. The cell consists of two mechanically coupled surfaces such that, when placed on a vibrating structure, the responses of the two surfaces are nearly out-of-phase and nearly of the same strength over a wide frequency range; i.e., acoustic dipole. The uniqueness of the proposed concept lies in the fact that the cell converts the motion of the vibrating structure into an inefficient radiating acoustic dipole source without the adverse effects introduced by active control. The control of low-frequency structurally radiated noise is then achieved by covering the structure with an array of these weak radiating cells, i.e., surface treatment. Thus the surface treatment essentially transforms the response of the structure to that of a distributed array of dipoles yielding a low sound radiating structure. A theoretical model of a single weak radiating cell applied to a simple piston-type structure is developed and verified analytically and experimentally.

\section{THEORY OF A WEAK RADIATING CELL}

At frequencies for which the acoustic wavelength exceeds the dimensions of a source, the volume velocity of the source essentially determines the radiated power. If the vol- 


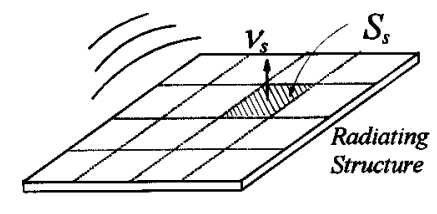

(a)

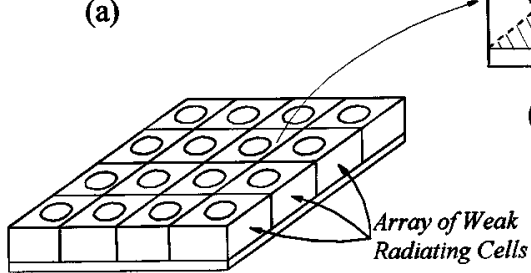

(c)

FIG. 1. Attenuation of structurally radiated sound using weak radiating cell concept: (a) untreated plate, (b) single cell, and (c) plate with array of cells.

ume velocity of the source is nearly zero, such as in a dipole, the radiated power is consequently minimized. ${ }^{18}$ Thus an efficient method to reduce the sound radiation from a structure would be to partition the surface of the structure into small sectors, each of which would independently respond as a dipole with zero volume velocity. To this end, the concept of a weak sound radiating cell is introduced to reduce the lowfrequency radiated noise from structures.

The weak radiating cell consists of two mechanically coupled surfaces such that, when placed on a vibrating structure, the responses of the two surfaces are nearly out-ofphase and of nearly the same strength over a wide frequency range. This leads the two surfaces to behave as an acoustic dipole and thus as a poor sound radiating source. Figure 1 illustrates the concept. A structure radiates sound into the surrounding fluid as shown in Fig. 1(a). The sound power radiated by a small area, $S_{s}$, with dimensions smaller than the acoustic wavelength, is determined by its volume velocity $Q_{s}=v_{s} S_{s}$, where $v_{s}$ is the average velocity over the small area. At low frequencies, the velocity of the area is nearly uniform and the area behaves as a monopole. Note that the total radiated power from the plate is due to the contribution of all the areas of the structure including their far-field coupling. Figure 1(b) illustrates a weak radiating cell covering the surface $S_{s}$. The concept of the weak radiating cell is to convert the motion of the small area from that of a monopole into a dipole source. To this end, the motion of the nearly uniform velocity $v_{s}$ of the surface $S_{s}$ is transformed into the motion of the two surfaces $S_{c 1}$ and $S_{c 2}$ with velocities $v_{c 1}$ and $v_{c 2}$, respectively. The cell is then designed such that the motion of these two surfaces are out-of-phase and of relative magnitude that leads to $v_{c 1} S_{c 1}+v_{c 2} S_{c 2} \cong 0$. Conceptually, by covering the structural surface with an array of cells as shown in Fig. 1(c), the response of the structure is converted to that of an array of dipoles, thus leading to an essentially zero volume velocity structural source and minimum sound radiation. It is important to reiterate that researchers have proposed active control methods to minimize the volume velocity of small panels. ${ }^{13-17}$ This purely active approach can have significant drawbacks that could limit its applicability in real systems. A more efficient

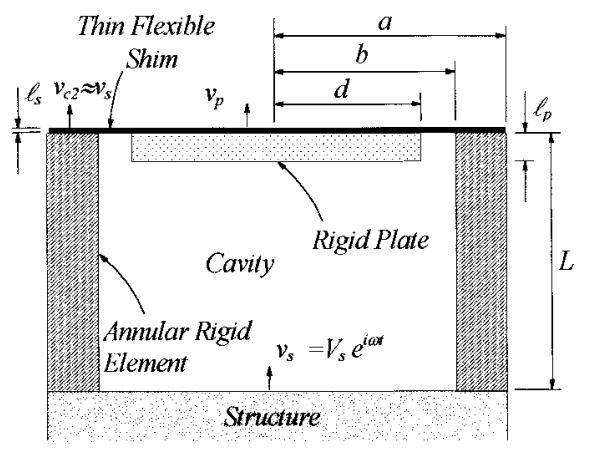

FIG. 2. Weak radiating cell implementation.

method would be to first obtain volume velocity cancellation passively, as proposed in this work, and then implement active control to further enhance the performance of the system if so desired. The hybrid approach is a matter of current research and will be reported in the near future.

An implementation of the weak radiating cell concept is illustrated in Fig. 2. Without loss of generality and for the sake of clarity in the presentation, the small area $S_{s}$ of the radiating structure is assumed to be a circular piston with uniform velocity, $v_{s}$. Hence for geometric consistency, the cell will also adhere to a circular configuration. Thus Fig. 2 shows a diametrical cross-section of the cell. The cell consists of a rigid annular element of area, $S_{c 2}$, which is mounted to the radiating structure surface. Because this element is assumed to be very stiff, the velocity of the surface $S_{c 2}$ is assumed to be same as the velocity of the structure, $v_{c 2} \cong v_{s}$. The second radiating surface of the cell, $S_{c 1}$, is obtained by attaching a rigid plate to the rigid element through a thin flexible shim which in turn creates a sealed cavity. The shim allows for stiffness in the radial direction with compliance in the axial direction. The mechanical behavior of the cell can be depicted as a single degree of freedom lumped parameter system $v_{p}$, i.e., motion of the rigid plate, with a base input $v_{s}$. Assuming harmonic input $v_{s}$ $=V_{s} e^{i \omega t}$, the response of the system $v_{p}=V_{p} e^{i \omega t}$ is given as ${ }^{19}$

$$
\left[c_{m}+i\left(\omega m_{p}-\frac{k}{\omega}\right)\right] V_{p}=\left(c_{m}-i \frac{k}{\omega}\right) V_{s},
$$

where $m_{p}$ is the system mass which includes both the mass of the rigid plate and the equivalent mass of the shim, $c_{m}$ is the shim damping, $k$ is the total stiffness of the system $k_{m}$ $+k_{f}=k$ with $k_{m}$ being the shim's mechanical stiffness and $k_{f}$ the stiffness due to the compressibility of the fluid in the cavity, and $V_{p}$ and $V_{s}$ are the complex velocity of the rigid plate and piston, respectively.

The complex source strengths for the original piston structure and the weak radiating cell can be obtained by integrating the surface velocity. The complex source strength of the original piston structure is simply $Q_{s}=S_{s} V_{s}$. The source strength of the cell is computed by integrating ${ }^{18}$

$$
Q_{c}=\int_{0}^{a} V(r) 2 \pi r d r
$$

where the radial complex velocity distribution $V(r)$ of the cell is defined as 

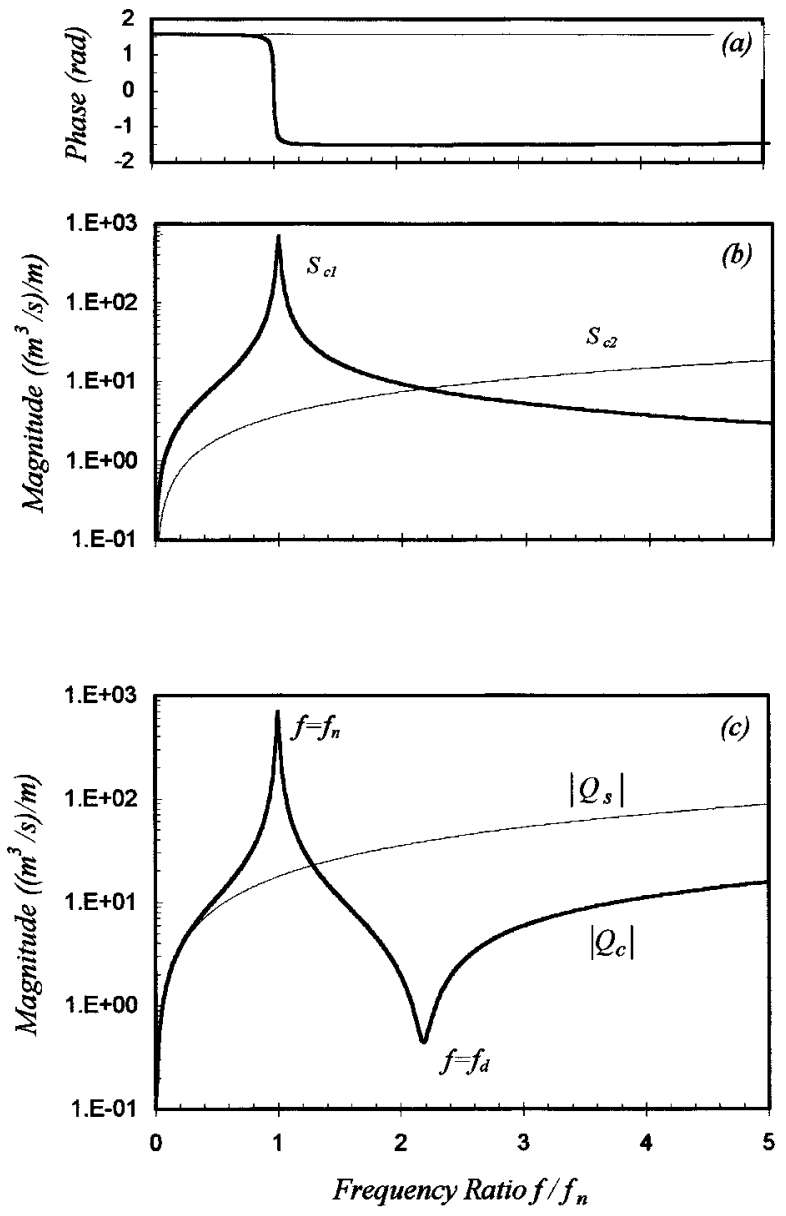

FIG. 3. Phase (a) and magnitude (b) of the source strength of surfaces $S_{c 1}$ and $S_{c 2}$, and magnitude (c) of source strength of weak radiating cell $\left|Q_{c}\right|$ and original structure $\left|Q_{s}\right|$.

$$
\begin{aligned}
& V(r) \\
& =\left\{\begin{array}{l}
V_{p}=\frac{c_{m}+k / i \omega}{i \omega m_{p}+c_{m}+k / i \omega}, \quad 0 \leqslant r \leqslant d \\
V_{h}(r)=\frac{V_{p}+V_{s}}{2}+\cos \left(\frac{\pi r}{b-c}\right) \cdot \frac{V_{p}-V_{s}}{2}, \quad d<r<b \\
V_{s}, \quad b \leqslant r \leqslant a,
\end{array}\right.
\end{aligned}
$$

which implies that the velocity profile of the shim is assumed to simulate a cosine function which varies $V_{p}$ at $r=d$ to $V_{s}$ at $r=b$, where $r$ is the radius from the center of the cell.

At low frequency where the acoustic wavelength $\lambda$ is much larger than the source dimension (i.e., $\lambda \gg a$ ), the acoustic power can be computed from the source strength as $^{18}$

$$
W=\frac{\alpha}{2} \pi \rho c\left(\frac{|Q|}{\lambda}\right)^{2}, \quad \lambda \gg a,
$$

where $\alpha=1$ for a free-field source and 2 for a baffled source, $\rho$ is the fluid density, $c$ is the speed of sound, and $\lambda$ is the acoustic wavelength.

For the sake of describing the general acoustic behavior of the cell, the magnitude and phase of the source strength of the two surfaces forming the cell are plotted in Figs. 3(a) and

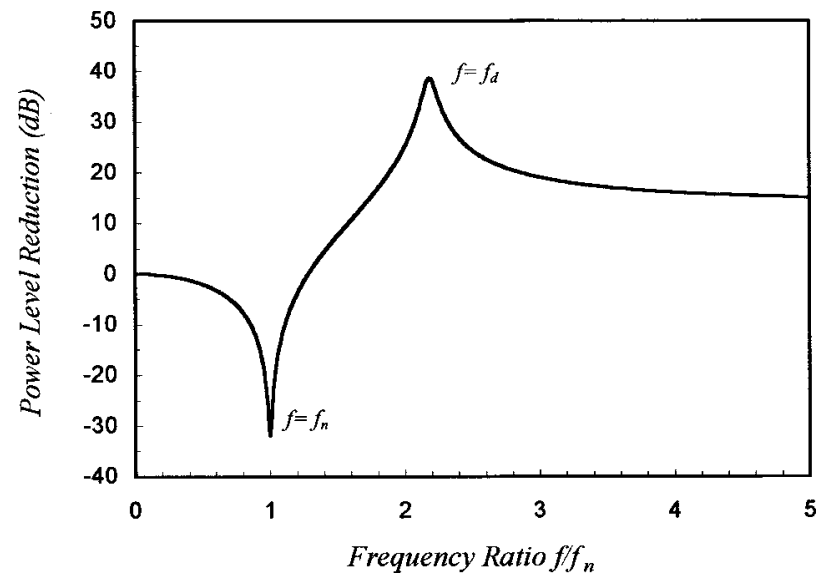

FIG. 4. Sound power level reduction achieved by the cell.

3(b). Moreover, the magnitudes of the source strength of both the cell and piston structure are shown in Fig. 3(c). These responses are plotted as a function of frequency normalized by the natural frequency of the plate-shim-cavity system, $f_{n}=1 / 2 \pi \sqrt{k / m_{p}}$. The sound power level reduction achieved by the cell as compared to the original piston is plotted in Fig. 4, again as a function of the normalized frequency. From Figs. 3(a) and 3(b), the general response of the cell can be described for the following frequency regions: below, around, and above the resonance of the plate-shimcavity system. It can be seen in Fig. 3(a) that, below the natural frequency of the system, the motion of the two surfaces is in phase and the sound radiated by the two surfaces adds to obtain the net radiation of the cell. At frequencies well below resonance, $f / f_{n} \leqslant 0.4$, the velocities of the two surfaces are nearly the same, and thus the cell radiates the same acoustic power as the original structure, and thus no power reduction is observed as seen in Fig. 4. When the frequency is around resonance, the plate response greatly exceeds that of the piston due to dynamic amplification, and the relative phase goes through a $180^{\circ}$ variation. The plate motion completely dominates the system response, resulting in an overall increase in the sound radiation from the structure. Above resonance, the motion of the two surfaces is out-of-phase and the radiation from the surfaces subtracts producing sound power reduction (see Fig. 4). Selecting proper areas will minimize the source strength of the resulting cell over a wide frequency range leading to the consequent sound reduction. From Figs. 3 and 4, it is clear that there is a frequency where the volume velocity $Q_{c}$ is a minimum and thus the sound reduction a maximum. Note that the volume velocity does not vanish at this frequency because the damping of the system does not result in a perfect outof-phase motion of the two cells' surfaces, i.e., the weak radiating cell behaves as a nearly perfect dipole. This frequency is referred as the cell's dipole frequency, $f_{d}$. To achieve optimal reduction in practice, the resonance of the system should then be placed below the frequency range of desired reduction and the dipole frequency where maximum sound reduction is desired. The control of tonal noise from transformers is a typical example where the cell should be designed so that the dipole frequency is tuned to the fre- 


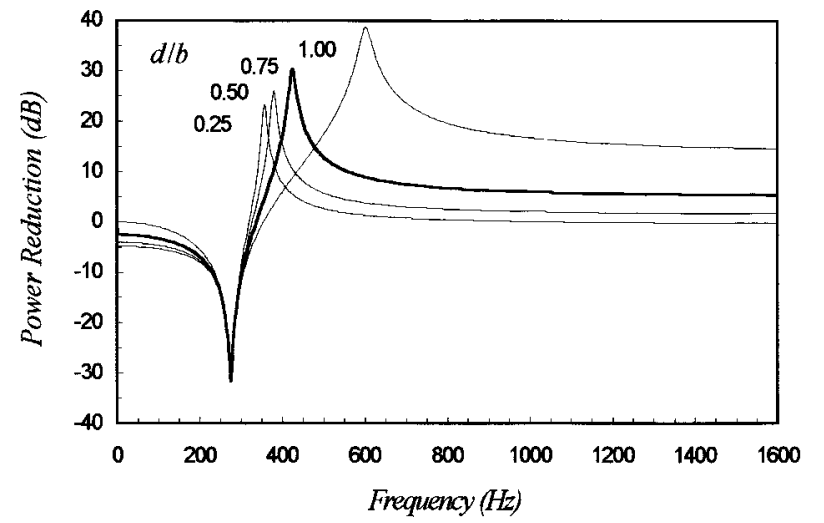

FIG. 5. Parametric study on $d / b$ ratio.

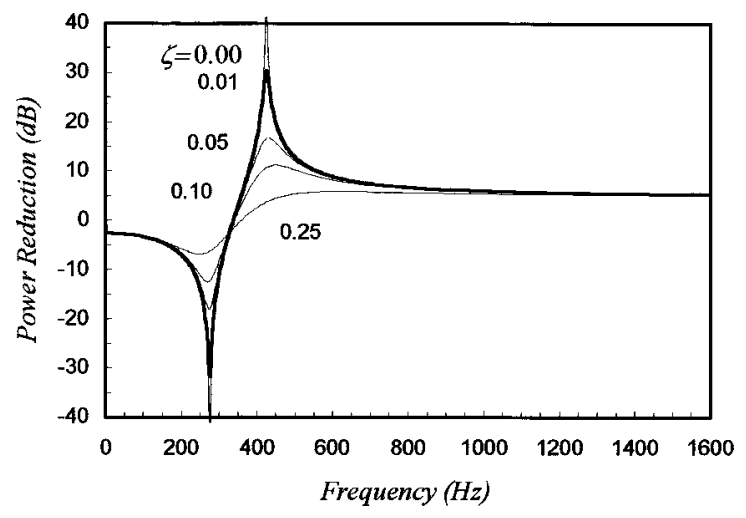

FIG. 7. Parametric study on damping ratio, $\zeta$. quency of the transformer noise tine, e.g., first harmonic of the line frequency $(120 \mathrm{~Hz})$. In Fig. 4, the increase in the sound power level is visualized at the resonance frequency, but more importantly, notice the region of maximum reduction around the dipole frequency where the source strength is minimized, i.e., a nearly perfect dipole is created with $38 \mathrm{~dB}$ of power reduction.

\section{PARAMETRIC STUDY}

The simplified model developed and properties used in the previous section served the purpose of introducing the concept of the weak radiating cell as well as to gain some insight into the behavior of the cell. In this section, parameters from realistic engineering materials will be used to study the system response and performance to assess the practicality of the concept. In addition, the influence on the cell performance to parameter variations will also be investigated. Here, the goal of the weak radiating cell is to reduce the sound radiation at frequencies below $1600 \mathrm{~Hz}$, and thus the cell is designed to this end.

Referring to Fig. 2, the rigid element is assumed here to be built from epoxy foam, commercially known as structural foam used in the automotive industry. ${ }^{20}$ The advantage of this material is its high modulus of elasticity $\left(E=642 \times 10^{6}\right.$ $\mathrm{Pa})$ and low density $\left(\rho=0.48 \mathrm{~g} / \mathrm{cm}^{3}\right)$ which is useful to minimize the overall weight of the cell which is a critical goal in some applications such as in aircraft interior noise. The dimensions of the rigid element are piston radius $a=57$ $\mathrm{mm}$, cavity radius $b=51 \mathrm{~mm}$, and cavity height $L=51 \mathrm{~mm}$.

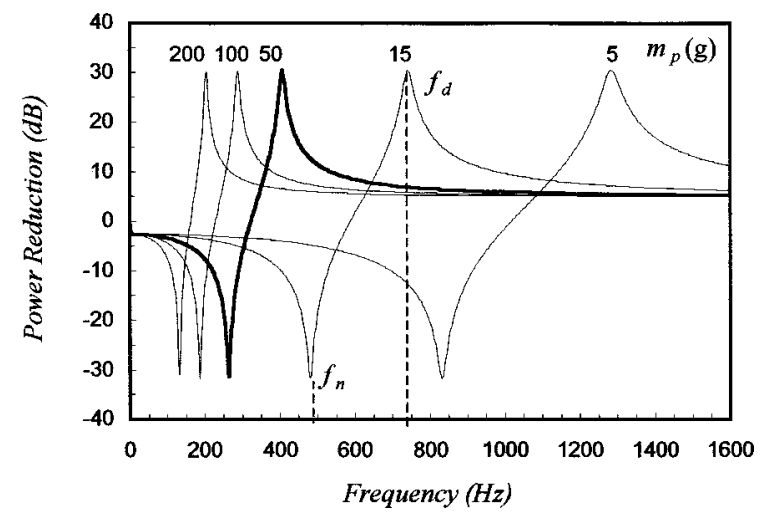

FIG. 6. Parametric study on system resonance, $f_{n}$.
The fundamental axial resonance of the rigid element was computed to be $3623 \mathrm{~Hz}$, which implies that this element behaves as rigid below $1600 \mathrm{~Hz}{ }^{21}$ The circular center plate forms the second surface. This plate should have the necessary mass for the desired dynamics of the cell and sufficient bending stiffness to essentially behave as a rigid plate in the frequency region of interest. Although a number of materials can be used, this plate is assumed here to be made of Plexiglas (modulus of elasticity $E=2758 \mathrm{Mpa}$, Poisson's ratio $\sigma=0.4$, and density $\rho=1300 \mathrm{~kg} / \mathrm{m}^{3}$ ) with a thickness of $l_{p}$ $=6.4 \mathrm{~mm}$ and a radius of $d=38 \mathrm{~mm}$. With these parameters, the fundamental bending plate resonance is $8811 \mathrm{~Hz}$, which is again outside the frequency range of interest. ${ }^{21}$ The flexible shim that attaches the center plate to the rigid element should be designed to be flexible in the vertical direction while providing some degree of stiffness in the plane normal to the intended motion. Here it is assumed that the shim is constructed of brass (modulus of elasticity $E=104 \mathrm{GPa}$, density $\rho=8500 \mathrm{~kg} / \mathrm{m}^{3}$, and loss factor $\eta=0.01$ ) with a uniform thickness of $l_{s}=0.2 \mathrm{~mm}$ and radius $a$.

The stiffness of the system has two components. Assuming clamped boundary conditions, the stiffness of the annular shim can be computed from the work by Leissa. ${ }^{22}$ In addition, the stiffness due to the fluid in the cavity is computed from the internal acoustic pressure. Assuming the fluid in the cavity is inviscid and the acoustic process is adiabatic, the stiffness due to the fluid in the cavity is given as ${ }^{18}$

$$
k_{f}=\frac{c^{2} \rho S_{c 1}^{2}}{V_{0}},
$$

where $\rho=1.21 \mathrm{~kg} / \mathrm{m}^{3}$ is the fluid density assumed to be air, and $c=343 \mathrm{~m} / \mathrm{s}$ is the speed of sound, and $V_{0}$ is the initial volume of the cavity.

Using the above materials and dimensions, the overall mass of the cell is $108 \mathrm{~g}$. The sound power level reduction of this baseline cell is plotted in Figs. 5-7 as a thick line. From these figures, the resonance frequency of the cell is $f_{n}$ $=276 \mathrm{~Hz}$ while the dipole frequency is $f_{d}=426 \mathrm{~Hz}$. Above the resonance of the cell, reduction is achieved starting at $338 \mathrm{~Hz}$ and then throughout the frequency band.

The first parameter investigated is the area ratio of the two radiating surfaces. This can be achieved by changing the ratio of the inner, $d$, to outer, $b$, diameter of the shim. It is assumed that the mass of the center plate does not change 


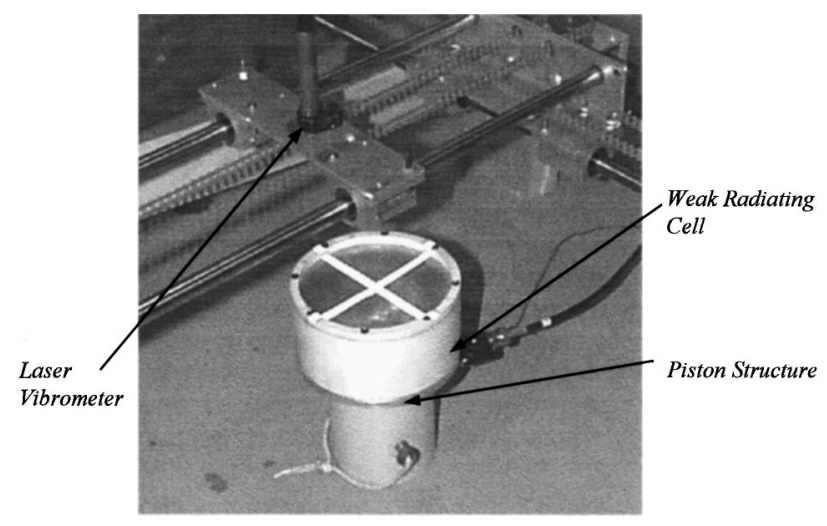

FIG. 8. Single weak radiating cell on a piston structure.

while the thickness of the shim is adjusted to keep the same natural frequency of the system. Figure 5 reveals that both the dipole frequency and the maximum reduction are sensitive to the area ratio which increases with the ratio $d / b$.

The next parameter to be investigated is the system resonance frequency. This can be altered by changing either the thickness or density of the plate to affect the mass and/or by changing the shim thickness to modify the stiffness. In this study, the shim thickness was held constant while the mass was changed. The resulting sound power reductions are shown in Fig. 6. As expected, the dipole frequency of maximum reduction occurs at lower frequencies as the mass is increased. However, the maximum level of power reduction is insensitive to the mass. Another interesting factor is the ratio of the dipole to the natural frequencies which decreases with the natural frequency of the system. Since it is important to minimize the overall weight of the cell for some applications, the mass should be minimized. This implies a minimization of the stiffness to yield a predetermined resonance frequency. The lowest stiffness is bounded by the size of the air cavity assuming a negligible contribution from the shim. For further reduction in the weight, the height of the cavity can be decreased. However, this increases the cavity stiffness. Note that as the size of the cell is proportionally reduced, the stiffness due to the fluid decreases in the same proportion as the cell size.

One approach to alleviate the increase in the radiated power at frequencies near the resonance is by introducing damping. Thus the effect of the shim-damping ratio on the system response is examined and shown in Fig. 7. As expected, increasing the energy dissipation leads to a reduction of the response at resonance. However, it also decreases the maximum sound reduction because it affects the relative phase between the motion of the two surfaces, i.e., the two surfaces are less out-of-phase as the damping increases. Consequently, there is a sound reduction trade off to introducing damping into the system.

\section{EXPERIMENTAL VALIDATION}

To verify the theoretical concept of a weak radiating cell, a cell was experimentally tested. The cell used for experimental verification possessed the dimensions and physical properties of the baseline cell analyzed in the numerical section, which is pictures in Fig. 8. The center mass was
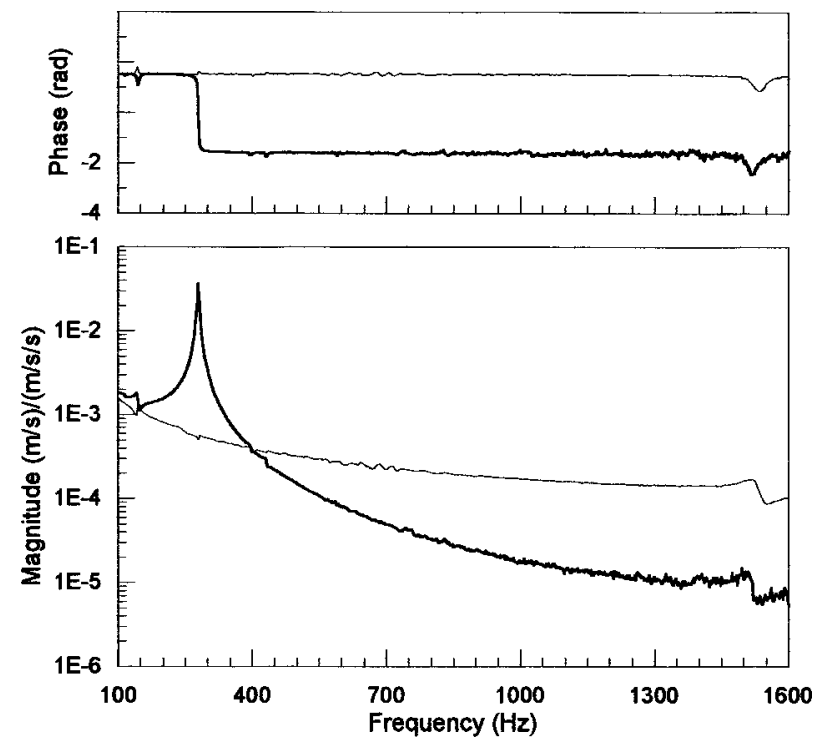

FIG. 9. Velocity response of inner (-) and outer (-) surfaces of weak radiating cell.

epoxied to the brass shim which was clamped to the rigid element using a thin ring of PVC and machine screws. The cell was then screwed to a rigid circular aluminum plate to ensure a tightly sealed cavity. The aluminum plate was mounted to a shaker and driven with white noise from 0 to $1600 \mathrm{~Hz}$. An accelerometer was positioned on the bottom side of the piston. The acceleration of the piston structure was used to normalize all the experimental data. The untreated piston structure, i.e., aluminum plate, is again used as a basis for the performance of the weak radiating cell. Both laser vibrometer scan and acoustic pressure measurements were taken to assess to dynamics and acoustic performance of the cell.

First, a laser vibrometer mounted to an automated traverse was utilized to scan the surface of the untreated piston and cell, which facilitated modal and source strength analysis (see Fig. 8). The velocity response, normalized by the piston acceleration, of both the inner plate and of the outer annular ring are presented in Fig. 9. The fundamental resonance of the cell occurs at $282 \mathrm{~Hz}$ where the phase goes through a $180^{\circ}$ variation. The mode shape is visualized in Fig. 10, which was obtained from a velocity scan across the surface of the cell. In this figure, the dashed lines indicate the location of the shim, i.e., $r=\mp b$ and $r=\mp d$. The second mode of the cell is a rocking motion that is not excited by the

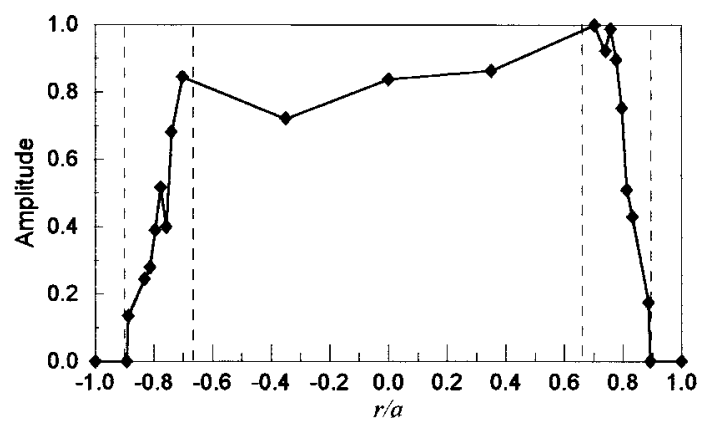

FIG. 10. Velocity profile across cell surface at $282 \mathrm{~Hz}$. 


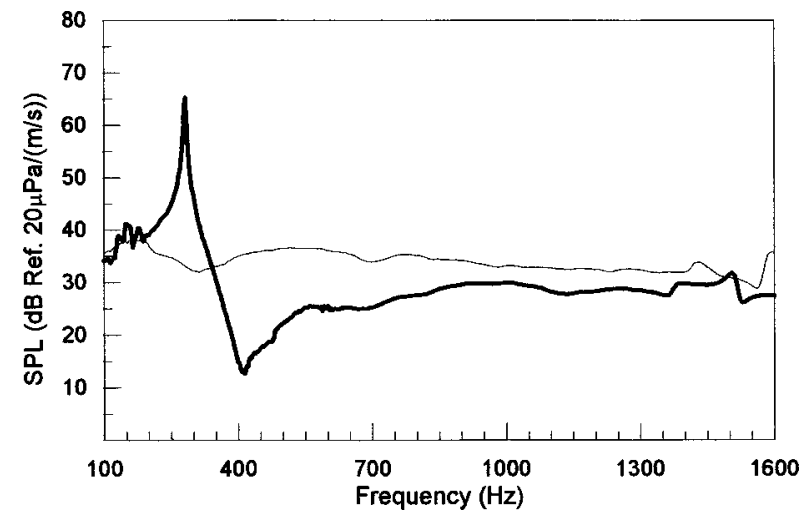

FIG. 11. Experimentally measured sound pressure levels due to piston (-) and cell (-).

uniform motion of the piston. It is important to remark that the presence of the rocking mode does not affect the system since it is itself a dipole source. The velocity response of the outer annular ring in Fig. 9 also shows that this element behaves as a rigid element in the frequency range $0-1600$ $\mathrm{Hz}$.

The second set of tests performed was pressure measurements. Both the original piston and the weak radiating cell were placed on a baffle such that both the cell and piston surfaces were flush to the baffle during their respective test cases. These experiments were performed in an anechoic chamber with a cutoff frequency of $250 \mathrm{~Hz}$. The sound radiation was then monitored with a microphone positioned 75 $\mathrm{cm}$ above the center of the systems. The sound pressure levels for each case are given in Fig. 11, which shows that the dipole effect of the cell produces a significant decrease in the pressure level above the resonance of the cell at the dipole frequency which occurs at $414 \mathrm{~Hz}$.

Since in the $0-1600 \mathrm{~Hz}$ frequency range of concern the acoustic wavelength is larger than the cell's dimension, the piston and cell are assumed to radiate as baffled simple sources. The acoustic power can then be computed using the pressure data as follows: ${ }^{18}$

$$
W=\frac{2 \alpha \pi R^{2} P^{2}}{\rho c}, \quad \lambda \gg a,
$$

where $R$ is the distance to the microphone from the source and $P$ is the pressure amplitude. The sound power level reduction computed from the pressure data is plotted in Fig. 12. There is an increase in the radiated power around the resonance of the cell. However, significant reduction levels are achieved throughout the frequency range above resonance, i.e., $f \geqslant 342 \mathrm{~Hz}$, with a maximum reduction of 22.9 $\mathrm{dB}$ at the dipole frequency $f_{d}=414 \mathrm{~Hz}$, i.e., the region of maximum reduction is where a nearly perfect dipole acoustic source is created. For comparison purposes, the analytically predicted sound power reduction is also plotted in Fig. 12, which shows very good agreement with the experimental results. A summary between experimental and analytical results is tabulated in Table I. The experimental values in this table were obtained from the experimental pressure data. Included in the table are the system resonance frequency, dipole frequency, and the sound power level reduction at the

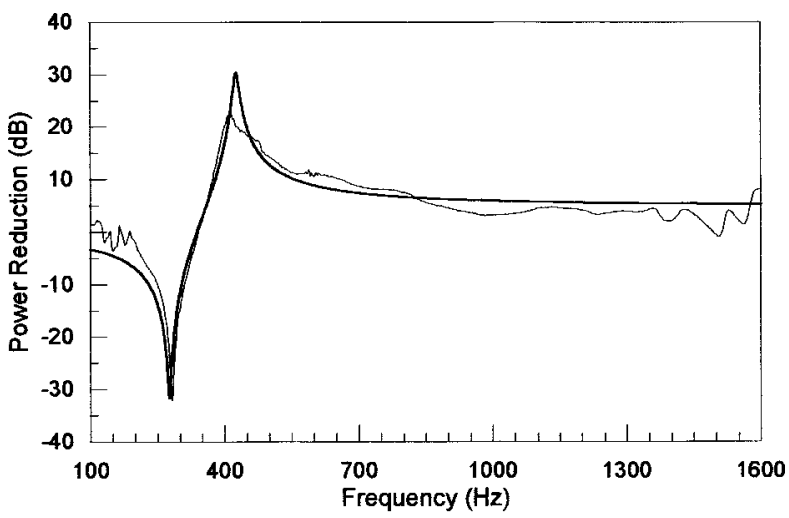

FIG. 12. Experimental (-) and analytical (-) sound power level reduction.

dipole frequency and in the frequency ranges $100-1600 \mathrm{~Hz}$ and $400-1600 \mathrm{~Hz}$. The $400-1600 \mathrm{~Hz}$ bandwidth is an important frequency range since both the theoretical model and experimental verification produces power reduction throughout this range. The resonance frequencies and overall power level reductions match very closely. The only discrepancy between the analytical and experimental values is in the reduction at the dipole frequency.

\section{CONCLUSIONS}

The concept of a weak radiating cell as a low-frequency passive control device for radiating structures has been introduced. In general, the concept converts the motion of a structure, assumed to radiate as a monopole, into an acoustic dipole source by creating two surfaces whose response is outof-phase and of nearly equal magnitude. Conceptually, applying an array of weak radiating cells to small partitions on a larger structure will create an array of distributed dipoles, thus minimizing the radiated sound from the structure. An analytical model was developed to predict the response and performance of a weak radiating cell applied to a simple piston structure. The reduction in sound power level between the piston and the piston with a cell applied was used to evaluate its performance. An overall power reduction of 6.7 $\mathrm{dB}$ between 400 and $1600 \mathrm{~Hz}$ was predicted with a maximum reduction of over $30 \mathrm{~dB}$ at the dipole frequency. A parametric study on the area ratio, system resonance frequency, and shim-damping ratio revealed important information for the optimum design of the cell.

Experiments to validate the concept of a weak radiating cell on a simple piston structure were performed. Pressure data were taken on a baffled weak radiating cell in an anechoic chamber while a laser vibrometer scan of the cell

TABLE I. Comparison of experimental and analytical results.

\begin{tabular}{|c|c|c|}
\hline Variable & Experimental & Analytical \\
\hline Resonance frequency, $f_{n}$ & $282 \mathrm{~Hz}$ & $276 \mathrm{~Hz}$ \\
\hline Dipole frequency, $f_{d}$ & $414 \mathrm{~Hz}$ & $426 \mathrm{~Hz}$ \\
\hline Sound power reduction at $f_{d}$ & $22.9 \mathrm{~dB}$ & $30.3 \mathrm{~dB}$ \\
\hline $\begin{array}{l}\text { Sound power reduction } \\
L_{w t}(100-1600 \mathrm{~Hz})\end{array}$ & $-8.1 \mathrm{~dB}$ & $-9.5 \mathrm{~dB}$ \\
\hline $\begin{array}{l}\text { Sound power reduction } \\
L_{w t}(400-1600 \mathrm{~Hz})\end{array}$ & $6.2 \mathrm{~dB}$ & $6.7 \mathrm{~dB}$ \\
\hline
\end{tabular}


surface allowed for modal and source strength analysis. An overall sound power level reduction of $6.2 \mathrm{~dB}$ was achieved between 400 and $1600 \mathrm{~Hz}$ with a maximum reduction of over $22 \mathrm{~dB}$ at the dipole frequency. These results closely match theoretical predictions and validate the potential of the weak radiating cell concept.

${ }^{1}$ B. S. Murray and J. F. Wilby, "Helicopter Cabin Noise-Methods of Source and Path Identification and Characterization," Proceedings of an International Specialist Symposium held at NASA Langley Research Center, Hampton, VA, May 22-24, 1978, NASA Conference Publication $2052(1978)$

${ }^{2}$ J. F. Wilby, "Aircraft interior noise," J. Sound Vib. 190, 545-564 (1996).

${ }^{3}$ K. Brungardt, J. Vierengel, and K. Wiessman, "Active Structural Acoustic Control of Noise from Power Transformers,' Noise-Con '97, Pennsylvania State University, June 15-17, 1997, pp. 173-182.

${ }^{4}$ R. A. Prydz, L. S. Wirt, H. L. Kuntz, and L. D. Pope, "Transmission loss of a multilayer panel with internal tuned Helmholtz resonators," J. Acoust. Soc. Am. 87, 1597-1602 (1990).

${ }^{5}$ J. Lee and G. W. Swenson, Jr., "Compact Sound Absorbers for Low Frequencies," Noise Control Eng. J. 38, 109-117 (1992).

${ }^{6}$ R. L. St. Pierre, Jr. and G. H. Koopman, "A Design Method for Minimizing the Sound Power Radiated from Plates by Adding Optimally Sized, Discrete Masses," Transactions of the ASME Special 50th Anniversary Design Issue, Vol. 117, pp. 243-251, June 1995.

${ }^{7}$ M. R. Jolly and J. Q. Sun, "Passive tuned vibration absorbers for sound radiation reduction from vibrating panels," J. Sound Vib. 191, 577-583 (1996).

${ }^{8}$ P. A. Nelson and S. J. Elliott, Active Control of Sound (Academic, San Diego, 1992).

${ }^{9}$ J. Aldrin, D. C. Conrad, and W. Soedel, "Investigation of passive and adaptive dynamic absorbers applied to an automatic washer suspension design," Proc. SPIE 2720, 50-61 (1996).

${ }^{10}$ M. A. Franchek, M. W. Ryan, and R. J. Bernard, "Adaptive passive vibration control," J. Sound Vib. 189, 565-585 (1995).

${ }^{11}$ S. A. Gentry, C. Guigou, and C. R. Fuller, "Smart foam for applications in passive-active noise radiation control," J. Acoust. Soc. Am. 101, 1771-1778 (1997).

${ }^{12}$ B. D. Johnson, "Broadband control of plate radiation using a piezoelectric, double-amplifier active-skin," Master's Thesis, Virginia Polytechnic Institute and State University, 1997.

${ }^{13}$ R. L. St. Pierre, Jr., W. Chen, and G. H. Koopman, "Design of Adaptive Panels with High Transmission Loss Characteristics," American Society of Mechanical Engineering, New York. Presented at the 1995 ASME International Mechanical Engineering Congress and Exposition, San Francisco, CA, November 12-17, 1995.

${ }^{14}$ K. Naghshineh and V. B. Mason, "Reduction of sound radiated from vibrating sources via active control of local volume velocity," Appl. Acoust. 47, 27-46 (1996)

${ }^{15} \mathrm{~T}$. W. Leishman and J. Tichy, "A fundamental investigation of the active control of sound transmission through segmented partition elements," Proceedings from Noise-Con 97 held at the Pennsylvania State University, Vol. 2, pp. 137-148, June 15-17, 1997.

${ }^{16}$ M. E. Johnson and S. J. Elliott, "Active control of sound radiation using volume velocity cancellation," J. Acoust. Soc. Am. 98, 2174-2186 (1995).

${ }^{17}$ S. J. Sharp, G. H. Koopman, and W. Chen, "Transmission loss characteristics of an active trim panel," Proceedings from Noise-Con 97 held at The Pennsylvania State University, pp. 149-160, June 15-17, 1997.

${ }^{18}$ L. E. Kinsler, A. R. Frey, A. B. Coppens, and J. V. Sanders, Fundamentals of Acoustics, 3rd ed. (Wiley, Canada, 1982).

${ }^{19} \mathrm{~W}$. T. Thomson, Theory of Vibration with Applications, 4th ed. (PrenticeHall, New Jersey, 1993).

${ }^{20}$ Novacore Structural Foam Brochure, Novamax Technologies, 13650 E. 10 Mile Road, Warren, MI.

${ }^{21}$ B. W. Ross, "Attenuation of structurally radiated noise with an array of weak radiating cells," Master's Thesis, Virginia Polytechnic Institute and State University (1997).

${ }^{22}$ A. Liessa, Vibration of Plates (Acoustical Society of America, New York, 1993), pp. 32-33. 